\title{
LA PARTICIPACIÓN SOCIAL PARA EL USO TURÍSTICO: CASO PARQUE NACIONAL ISLAS MARIETAS, MÉXICO
}

Social Participation for Tourist Use: The Case of Parque Nacional Islas

Marietas, Mexico

Myrna Leticia Bravo Olivas ${ }^{1}$
Rosa María Chávez Dagostino ${ }^{2}$
José Luis Cornejo Ortega $^{3}$
Recibido: mayo, 2018 // Aceptado: mayo, 2018

RESUMEN

El uso público de las áreas naturales protegidas incluye los usos turístico, educativo, interpretativo, recreativo y de investigación, realizados por visitantes que participan en actividades que no extraen ni introducen elementos ajenos al área. El objetivo de esta investigación fue establecer una visión colectiva sobre las condiciones sociales y biofísicas deseadas para el Parque Nacional Islas Marietas, México. Esto se construyó mediante talleres participativos con actores clave, para promover el diálogo entre cerca de 200 participantes a través de la herramienta World-Café. Se discutió la problemática de la visitación y actividades recreativas. Establecieron las inquietudes y oportunidades, luego emitieron sus conclusiones con respecto a la problemática que jerarquizaron de acuerdo a su importancia y se consensaron. La problemática identificada y jerarquizada con respecto a la visitación del parque fue: concentración de visitantes en un mismo lugar que rebasa la capacidad de carga y disminuye la calidad de la experiencia; que, aunque los prestadores de servicios turísticos han mejorado su actitud, existen malas prácticas y violaciones a las reglas; que la promoción del parque es inadecuada; que la vigilancia es insuficiente; que las zonas frecuentemente visitadas han disminuido su atractivo para el buceo autónomo y se han deteriorado las comunidades coralinas en la zona. Con esta información se estableció una línea base socialmente acordada para el

\footnotetext{
${ }^{1}$ Doctor en Ciencias. Universidad de Guadalajara. Líneas de investigación: Prospectiva, desarrollo, patrimonio y turismo. Dirección: Av. Universidad 203, Delegación Ixtapa C.P. 48280, Puerto Vallarta, Jalisco México. Teléfono: 3222262200 ext. 66305. E-mail: myrna.bravo@cuc.udg.mx

${ }^{2}$ Doctor en Ciencias. Universidad de Guadalajara. Líneas de investigación: Prospectiva, desarrollo, patrimonio y turismo. Dirección: Av. Universidad 203, Delegación Ixtapa C.P. 48280, Puerto Vallarta, Jalisco México. Teléfono: 3222262200 ext. 66305. E-mail: rosac@cuc.udg.mx

${ }^{3}$ Doctor en Ciencias Universidad de Guadalajara. Líneas de investigación: Prospectiva, desarrollo, patrimonio y turismo. Dirección: Av. Universidad 203, Delegación Ixtapa C.P. 48280, Puerto Vallarta, Jalisco México. Teléfono: 3222262200 ext. 66305. E-mail: Jose.cornejo@cuc.udg.mx
} 
manejo del área, proceso que constituye la base para el uso público que todavía está en proceso y donde participan diversos actores.

Palabras clave: uso público, condiciones sociales/biofísicas, área natural protegida.

\section{ABSTRACT}

The public use of natural protected areas include tourist, educational, interpretive, recreational and research uses performed by visitors who participate in nonextractive activities nor introduce strange elements in the area. The objective of this research was to establish the desired social and biophysical conditions for Parque Nacional Islas Marietas, Mexico. The research was carried out through participatory workshops using World-Café tool to promote dialogue among some 200 participants. The problem of visitation and recreational activities were discussed. They established concerns and opportunities, and then issued their conclusions regarding the problems that were ranked according to their importance, which were agreed upon by all. The problems that were identified and hierarchicly organized with respect to the visitation of the park through social participation were the following: concentration of visitors in the same place that exceeds the carrying capacity and reduces the quality of the experience; although tourism service providers have improved their attitude, there are still bad practices and violations of the rules; the promotion of the natural area is inadequate; surveillance is insufficient; the areas frequently visited have diminished their appeal for scuba diving and coral communities have been deteriorated. On this basis, the baseline for managing the area was socially established, a process that constitutes the basis for public use that is still in process, where different actors participate.

Key words: Public Use, Social/Biophysical Conditions, Natural Protected Area.

\section{INTRODUCCIÓN}

El fortalecimiento de vínculos y la cooperación entre Latinoamérica y el Caribe ha traído aprendizajes que permiten mejorar las tareas de conservación, entre ellas la gestión del turismo en las áreas naturales protegidas (ANP). La colaboración entre diversos actores autoridades de gobiernos locales y nacionales, los profesionales de la conservación y del turismo, prestadores de servicios y visitantes - ha permitido reflejar las oportunidades que brinda el turismo para mejorar la calidad de vida de las poblaciones locales y conservar, al mismo tiempo, la diversidad biológica en ecosistemas únicos de las áreas naturales, generando un valor a través de una actividad económica viable e inclusiva, donde se ha propuesto al ecoturismo como eje central (Londoño, 2017). 
Antes de la década de 1980, las comunidades tendían a ser excluidas sistemáticamente en la toma de decisiones (Halfter, 1984; PazSalinas, 2008) e incluso su participación era considerada contraproducente para el manejo de los recursos naturales (Ruíz-Mallén et al. 2013). Posteriormente este enfoque fue desafiado por diversos estudios que hicieron hincapié en los derechos y la necesidad de la participación local en la toma de decisiones en áreas protegidas, promovido fuertemente en sucesivos congresos mundiales sobre parques nacionales (Calvet-Mir, Maestre-Andrés \& van den Bergh, 2015), durante más de una década.

Otro punto de debate con respecto a las ANP es la percepción dual que existe: como áreas de oportunidad para el desarrollo local-regional donde el turismo juega un papel importante o, como zonas que limitan el acceso a mejores condiciones de bienestar para la población, cuando se aíslan de la comunidad local en aras de la conservación (Naughton-Treves, Buck Holland \& Brandon, 2005).

Por otra parte, el papel de las ANP ha sido cuestionado en varios sentidos, uno de ellos, su impacto en el desarrollo local, cuando no existe un cambio significativo en la mejora de condiciones en las comunidades asociadas a esas áreas. Riemann, Santes-Álvarez y Pombo (2011) propusieron dar seguimiento a este aspecto para reenfocar las actividades de conservación de los recursos, bajo una nueva perspectiva que incluya el beneficio social y no solo lo suponga.

Recientemente, la participación activa de los diversos actores ha sido reconocido como un factor clave de la protección efectiva de las áreas naturales, para el manejo de los recursos naturales y la conservación de la biodiversidad (Borrini-Feyerabend et al., 2013). Para estos efectos, participación será cualquier tipo de inclusión de actores no estatales: miembros del público en general o partes interesadas organizadas, en cualquier etapa del establecimiento de la política gubernamental (Wesselink et al., 2011). La participación social es un derecho ciudadano referido a iniciativas de la sociedad donde los individuos son conscientes de un hecho en un espacio determinado, y se agrupan para realizar tareas y defender determinadas causas demandando cambios, hechos que requieren del manejo de estructuras sociales de poder.

El logro de objetivos consensuados tiene sentido desde lo colectivo, revelando intereses, miedos o aspiraciones de los involucrados. La participación efectiva supone también condiciones de igualdad y 
requiere de sujetos participantes informados, lo que puede ser una limitante en un principio (Espinosa, 2009).

Según Calvet-Mir, Maestre-Andrés y van den Bergh (2015), en este siglo se ha enfatizado la necesidad de participación social en decisiones gubernamentales por varias razones: se aseguran decisiones más legítimas, mejorando así la credibilidad pública en los gobiernos; reduce los conflictos potenciales entre los diferentes interesados; aumenta la variedad de información que contribuye a mejores decisiones; y contrarresta el poder de los intereses establecidos al permitir que todos los afectados influyan en el proceso de decisión asociado.

El papel de las autoridades para hacer que la participación social sea efectiva es determinante, sin embargo, no siempre cumple con la inclusión de diversos actores sociales en la instrumentación de políticas o, con el fin de “incrementar la legitimidad” (Patten, 2000).

Así, las áreas naturales deben ser administradas como un continuo de vida que desconoce límites políticos y administrativos de tal forma que, la dinámica social donde se incluyen una serie de necesidades sociales y económicas, debe orientar el futuro de una ANP, considerando los impactos ambientales directos que se pueden provocar por el uso público en general o por sus efectos secundarios (Rodríguez \& Bracamonte, 2008).

El análisis del fenómeno turístico asociado a las ANP ha sido abordado con una intensidad creciente debido al incremento en la oferta y la demanda de este tipo de actividades, además de la problemática derivada, donde su gestión y la participación social sigue siendo un reto.

En este trabajo se presenta un caso único en México, considerando, por una parte, los problemas derivados de una creciente visitación y gestión del uso público, y por la otra, la participación social resultante en el Parque Nacional Islas Marietas (PNIM). El objetivo de esta investigación fue establecer las condiciones sociales y biofísicas deseadas (visión), como base para el uso público del PNIM, a través de un ejercicio de participación social y su resultado. Para ello se explica a continuación la problemática y conflictos que hicieron necesaria la participación social formal y las características del área natural, con el fin de contextualizar el ejercicio. 


\section{CARACTERÍSTICAS DEL ÁREA DE ESTUDIO}

Según la Comisión Nacional de Áreas Protegidas en México, el PNIM tiene una superficie total de 1.383,95 hectáreas, su parte insular abarca 78,94 hectáreas y la superficie restante corresponde al área marina (CONANP, 2005, p. 9). Se localiza en la Bahía de Banderas en el Pacífico mexicano, a $6 \mathrm{~km}$ hacia el suroeste de la península de Punta de Mita, municipio de Bahía de Banderas, Nayarit (Figura 1). Incluye las islas Larga y Redonda además de otros islotes, que forman parte del sistema turístico de Puerto Vallarta y la Riviera Nayarit. Desde el punto de vista biogeográfico, el Parque se encuentra ubicado en la zona de transición entre las regiones neártica y neotropical, por lo cual, tanto en tierra como en la zona marina, cohabitan especies que se encuentran en los límites de su respectiva distribución. Asimismo, se localizan en una zona de confluencia de tres masas de agua: la Corriente de California, la Corriente Costera de Costa Rica y la masa de agua del Golfo de California (Wyrtki, 1965, p. 272).

Figura 1. Localización de PNIM.

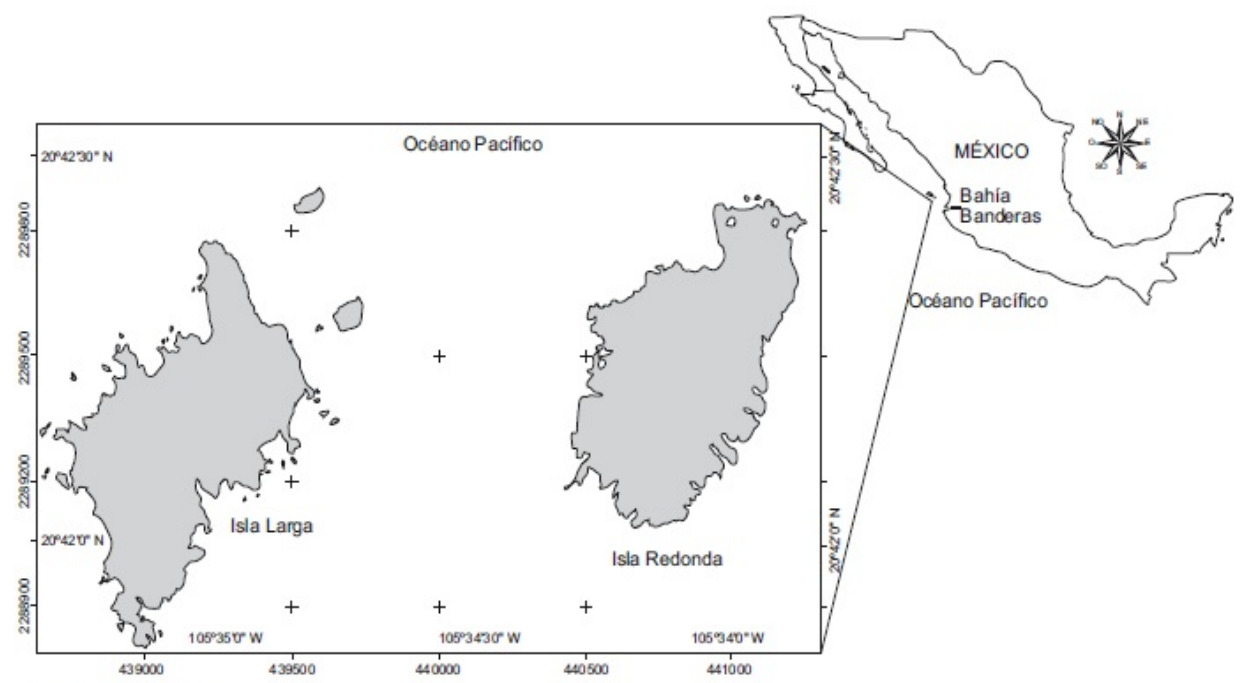

Fuente: Cornejo-Ortega \& Chávez Dagostino (2014). 
El Parque posee una particular belleza escénica natural y diversidad biológica reconocida como zona prioritaria para la conservación a través de distintas nominaciones: Áreas de Importancia para la Conservación de las Aves en México (AICAS) con la categoría G-4-A (AICA 29); Sitio Ramsar 1345 (desde el 2004) y Reserva de la Biósfera del Programa el Hombre y la Biósfera de la UNESCO (inscrita en el 2008).

Con respecto a las islas principales que conforman el Parque, la isla Larga mide poco más de un kilómetro de longitud y 800 metros de ancho en su porción occidental. Tiene algunas playas rocosas protegidas por acantilados que llegan hasta los 35 metros de altura. Su máxima altura sobre el nivel del mar es de 43 m (Gaviño \& Uribe, 1981). Se pueden observar formaciones geológicas que originan bufadoras y arcos rocosos debajo de los cuales se produce fuerte oleaje. La isla Redonda, por su parte, se localiza al este de la isla Larga, con bordes marinos en forma de acantilados abruptos con una altura máxima de 40 metros, no presenta playa alguna para un desembarco adecuado.

El Parque cuenta con cuatro zonas núcleo y una zona de amortiguamiento. Dentro de estas se han establecido subzonas de Protección (UPr), Uso Restringido (UR), de Aprovechamiento Sustentable de los Recursos Naturales, de Uso Público (UP) y de Recuperación. En el cuadro 1 se muestran las subzonas que contienen los 10 sitios de uso público del PNIM (CONANP, 2005).

Cuadro 1. Zonas, subzonas y sitios de uso público. UR - Uso

Restringido, UP - Uso Público

\begin{tabular}{|c|c|c|}
\hline Zona & Subzona* & Sitios \\
\hline \multirow{3}{*}{ Núcleo } & UR1 & Sendero terrestre \\
\cline { 2 - 3 } & UR2 & Escalones de Piedra \\
\cline { 2 - 3 } & UR3 & Playa del Amor \\
\hline \multirow{4}{*}{ Amortiguamiento } & UP1 & Cueva del Muerto \\
\cline { 2 - 3 } & UP2 & La Nopalera \\
\cline { 2 - 3 } & UP3 & Pared \\
\cline { 2 - 3 } & & Túnel-Amarradero \\
\cline { 2 - 3 } & & Plataforma Pavona \\
\cline { 2 - 3 } & Zona de Amortiguamiento & Bajo rocoso \\
\cline { 2 - 3 } & & General \\
\hline
\end{tabular}

Fuente: Elaboración propia con base a datos de CONANP (2005). 


\section{PROBLEMÁTICA SOBRE EL MANEJO DEL PARQUE}

Aunque el decreto del PNIM se dictó en el 2005, el uso público no había representado problema. En 2014 la CONANP, a través de un estudio técnico que consideró la información disponible sobre las condiciones de fragilidad y resiliencia de la biodiversidad, los impactos de los visitantes, los resultados del manejo de los mismos y las condiciones del entorno, determinó las intensidades de uso (Biósfera, 2014).

Recientemente, el análisis del fenómeno turístico en el PNIM ha sido abordado con mayor intensidad por el incremento en la afluencia de visitantes y la problemática asociada. Por ejemplo, se calculó la capacidad de carga turística del sendero terrestre de la isla Larga (Cornejo-Ortega, Chávez-Dagostino \& Cupul Magaña, 2011) e hicieron algunas consideraciones para la evaluación del impacto ambiental del buceo recreativo en el Parque (Preciado, Simental, Ochoa-Chávez \& ChávezDagostino, 2011). También se evaluó el éxito reproductivo de los pájaros bobos en la isla Larga como indicador de perturbación por uso turístico (Cornejo-Ortega, Chávez-Dagostino \& Cupul Magaña, 2016).

La capacidad de carga para el buceo libre y buceo autónomo en sitios de uso público del PNIM fue analizado en el 2014 (Biosfera, 2014: 39). En este contexto, se realizó este trabajo durante el 2015 y parte del 2016, dando inicio a nuevos debates y una reordenación todavía en proceso. Posteriormente, Cupul-Magaña y Rodríguez (2017, p. 1) recalcularon la capacidad de carga efectiva de actividades de buceo, resultado para el buceo SCUBA 22.4-46.4 buzos al día, mientras que para el snorkel se estableció entre 83-46.4 buzos libres por día.

Dado que el uso público es dinámico y no existían antecedentes referentes a una línea base de los ecosistemas protegidos o caracterización de los visitantes y sus impactos en los sitios de uso público, resultaba urgente su ordenamiento en el Parque.

La problemática más evidente del uso público en el PNIM se circunscribe a la porción marina del Parque debido al incremento de la cantidad de visitantes cada año, puesto que en la superficie terrestre no están permitidos los visitantes, excepto en un sendero en la isla Larga. Las embarcaciones turísticas y los visitantes de las islas se concentraban en su mayoría en los mismos sitios y a las mismas horas del día, especialmente, la Playa del Amor o Playa Escondida, con el consecuente riesgo de 
impacto sobre los ecosistemas marinos. Sin embargo, la visión de los diferentes actores era divergente con respecto a las causas o los impactos negativos.

Otro reto fue la gestión de la demanda exponencial de visitantes. En el año 2012, el número de visitantes registrados por la Dirección del PNIM fue de alrededor de 27500 visitantes, mientras que en 2013 aumentó a 49765 y para octubre de 2014 prácticamente se duplicó el número de visitantes registrados en comparación con el año 2012, la mayor parte concentrados en la Playa del Amor y su zona de acceso, excediendo significativamente la capacidad de carga de este sitio que es de 95 visitantes/día (Biósfera, 2014, p. 21).

El uso de la biodiversidad en las ANP en México es ordenado a través de los programas de uso público. De acuerdo con la Estrategia Nacional para un Desarrollo Sustentable del Turismo y la Recreación en las Áreas Naturales Protegidas de México (2012), el uso público de las ANP incluye los usos turístico, educativo, interpretativo, recreativo y de investigación, realizados por visitantes que participan en actividades que no extraen (excepto para el deporte, la investigación y monitoreo) ni introducen recursos en un área natural o cultural protegida (CCAD \& SAM, 2005, p. 7).

La problemática para la conservación del PNIM y la generación de servicios socio-ambientales se derivan básicamente de dos aspectos: la influencia que ejerce el contexto turístico regional y las afectaciones causadas por las actividades dentro del Parque y en su área de influencia inmediata. En este contexto, era necesaria su caracterización y ordenamiento.

Considerando que toda actividad humana produce un impacto de distintas magnitudes, las decisiones sobre el uso que se emprenda en estas áreas de alta diversidad y vulnerabilidad requieren un análisis participativo de costo-beneficio, donde pueden aceptarse estos usos con ciertos límites, generando impactos mínimos y desde esta perspectiva, las áreas naturales pueden cumplir de mejor forma su cometido de conservación, por tanto la participación y acuerdo de los principales actores es determinante. 


\section{METODOLOGÍA}

Las condiciones sociales y biofísicas deseadas se construyeron a través de tres talleres participativos para la elaboración del programa de uso público del PNIM (2015), en las localidades de Nuevo Vallarta y Cruz de Huanacaxtle, Nayarit, y en Puerto Vallarta, Jalisco. Además, se integró el estudio previo de Biósfera (2014) relacionado con el uso público. Se utilizó la técnica de World Café para promover el diálogo entre los cerca de 200 participantes con diverso perfil: prestadores de servicios, sectores de gobierno, turismo, medioambiente, académicos, marina, protección civil, sociedad civil organizada, pescadores, entre otros, los cuales trabajaron en cuatro mesas propuestas: Visitación, Zonificación, Reglamentos y Participación Social.

La herramienta de World Café (café del mundo) es un proceso de conversación humana, cálida y significativa que permite a un grupo de personas dialogar sobre preguntas poderosas, para generar ideas, acuerdos y caminos de acción creativos e innovadores, en un ambiente acogedor y amigable, semejante al de una cafetería. Esta metodología fue desarrollada por dos consultores mexicanos: Juanita Brown y David Isaacs y ha sido utilizada desde el 2005 en los más variados escenarios, con diferentes grupos de edad, con diferentes culturas, para propósitos diversos en diversas partes del mundo.

Este proceso se fundamenta en dos principios:

- $\quad$ Primero: los seres humanos queremos hablar juntos de las cosas que nos son importantes,

- $\quad$ Segundo: a medida que hablamos juntos, podemos tener acceso a una sabiduría superior, que solo se encuentra en la colectividad (Brown \& Isaac, 2005).

De manera general, se desarrolla mediante conversaciones en mesas de cuatro a seis personas, que de manera simultánea analizan un tema o pregunta durante un tiempo determinado. Al final de cada pregunta los participantes cambian de mesa y continúan la discusión con otros participantes y así sucesivamente hasta abordar todos los temas propuestos.

Previo al ejercicio se explicaron los objetivos y se expusieron temas sobre la conservación y el uso público de las ANP, por parte de la 
Directiva del PNIM. Luego se discutió la problemática de la visitación y actividades recreativas.

Para la integración de las mesas se formaron equipos tomando en cuenta la participación de al menos un representante de cada sector en cada mesa distribuidos en cuatro ejes: Visitación, Zonificación, Reglamentos y Participación Social. En cada una se plantearon dos preguntas básicas (ver Cuadro 3). A partir de estas preguntas, los integrantes del equipo analizaron la problemática, escribieron sus principales conclusiones, establecieron las inquietudes y oportunidades, que luego compartieron con respecto a la problemática que jerarquizaron de acuerdo a su importancia y se consensuaron. Al concluir, el equipo rotaba hacia la siguiente mesa y repetían el ejercicio.

La información fue consensuada en un debate final e integrada por un comité y consultada públicamente por diferentes medios. Luego se convocó a prestadores de servicios turísticos (PST) y pescadores, se socializaron las estrategias propuestas en las mesas de trabajo y estableció la pertinencia de cada una, el plazo en el que debía ser establecida y el órgano responsable de aplicar la estrategia.

Estos resultados se integraron a la propuesta del Plan de uso público del PNIM, actualmente en proceso.

\section{RESULTADOS}

La capacidad de manejo del Parque tiene puntos débiles a consecuencia de la escasez de recursos físicos (equipo e infraestructura) y humanos (personal) que impiden un rendimiento óptimo de las funciones, la administración y, sobre todo, el monitoreo diario de los visitantes. Un ejemplo de lo anterior sucedió en 2012, cuando una fotografía de Playa del Amor circuló profusamente en las redes sociales, acarreando un incremento descontrolado de visitas al sitio, que en temporada alta llegó a recibir hasta 3000 personas por día, pero sin lograr disfrutar su belleza escénica y causando daños al ecosistema del sitio.

Si bien existe una colaboración por parte de algunos PST en las acciones de vigilancia, el apoyo de estos resulta indispensable, además de implementar un buen uso de los sitios de buceo. La administración del Parque no cuenta con los recursos suficientes para fortalecer las capacidades y herramientas necesarias (personal y equipos suficientes capacitación acorde a las necesidades de manejo del área), para lograr una 
efectiva colaboración entre usuarios y autoridades en la ejecución de este Programa de Uso Público. De los trabajos realizados en el taller se desprende la importancia de las actividades que en la actualidad se realizan en el PNIM, que a continuación se describen.

\section{TIPIFICACIÓN DE LAS ACTIVIDADES DE USO PÚBLICO}

BUCEO AUTÓNOMO: es el acto por medio del cual una persona se sumerge completamente en cuerpos de agua. Para realizar esta actividad se necesita equipo especializado: máscara, traje de buceo, aletas, cinturón de lastre, equipo autónomo SCUBA, tanque de aire comprimido, chaleco hidrostático, regulador, reloj profundímetro, manómetro. La duración de esta actividad es de 6 horas.

BUCEO LIBRE: es la técnica conocida como “a pulmón“, es una manera sencilla de apreciar la flora y fauna marina, para lo cual solo se necesita: máscara, traje de buceo, chaleco y aletas. La duración de esta actividad es de 2 horas.

OBSERVACIÓN DE FLORA Y FAUNA Y RECORRIDO TURÍSTICO: Se refiere a los viajes comerciales que permiten a los turistas observar o escuchar cualquiera de las especies de ballenas, delfines o marsopas (cetáceos), aves y flora existente en su hábitat natural. El equipo que generalmente se necesita para esta actividad es una cámara fotográfica para captar la experiencia en imágenes. De igual forma se puede realizar el recorrido turístico en barco para observar el paisaje completo de las islas. El tiempo de duración oscila entre 4 y 8 horas.

DESEMBARQUE EN PLAYAS: consiste en bajar a la playa y tomar el sol, solo se necesita ropa de playa y toalla. Con una duración de media hora en la Playa del Amor y en la Playa La Nopalera.

KAYAK: es un deporte acuático, en el que se va sentado mirando hacia la parte delantera (proa), en el sentido de la marcha. Se palea en un kayak, y consigue el desplazamiento en el agua (propulso) con la ayuda de la pala. La duración de la actividad oscila entre una y tres horas.

PADDLE BOARD: El surf de remo es una forma de deslizamiento en la que el navegante utiliza un remo para desplazarse por el agua mientras permanece de pie en una tabla de surf. La duración de la actividad oscila entre una y tres horas. 
NATACIÓN: es el movimiento y el desplazamiento a través del agua mediante el uso de las extremidades corporales y por lo general sin utilizar ningún instrumento o apoyo para avanzar, generalmente la natación se hace para recreación, deporte, ejercicio o supervivencia. Esta actividad tiene una duración de media hora.

\section{LOS PROVEEDORES DE SERVICIOS TURÍSTICOS}

Para 2016 en el padrón pst se encontraban registrados 173 dedicados principalmente a cinco actividades en el pnim: recorrido turístico, buceo libre, buceo autónomo, observación de flora y fauna y desembarque en playas. cuentan con una oferta total de 3172 espacios para los turistas, 96 tienen embarcaciones con más de 10 espacios y 77 ofertan menos de 10 espacios, 83 fueron nuevas autorizaciones y 90 fueron prórrogas de permisos en 2015.

En este sentido, el uso público del parque ha sido fundamental para los pst que se encuentran en la localidad de punta de mita, considerada la población directamente relacionada con el pnim.

A raíz del cierre temporal de la playa del amor en el 2016, surgieron conflictos entre la cooperativa corral del risco (punta de mita), la asociación de embarcaciones turísticas de puerto vallarta y las autoridades del Parque, tanto por las pérdidas económicas como por la molestia de los turistas que ya tenían reservado su paseo y tuvieron que cancelar la visita. Así lo expresó el Presidente de la Asociación de Embarcaciones Turísticas de Puerto Vallarta, además explicaron que cada semana serían un total aproximado de 2500 turistas los que podrían dejar de visitar la Playa del Amor, entre los 178 lancheros que tienen sus respectivos permisos de la Secretaría de Medio Ambiente y Recursos Naturales (SEMARNAT).

Los PST reconocen que:

- El objetivo del ANP es conservar, proteger, recuperar los ecosistemas mediante implementación de acciones de manejo y administración del Parque Nacional con la participación que corresponde al sector gobierno, académicos, prestadores de servicios y sociedad en general.

- Constituyen un bien del patrimonio natural de la humanidad reconocido por la convención de la UNESCO.

- Abarca zona terrestre y marina con una superficie de más de 1.383,95 hectáreas. 
- Presenta una zona núcleo con zonas de uso restringido y zonas de protección y una zona de amortiguamiento que sostiene zonas de uso público y de aprovechamiento sustentable de recursos naturales.

- Existe actualmente un monitoreo tanto de aspectos físicos y biológicos como de visitantes en distintos sitios de las islas generando información de calidad que permite una mejor toma de decisiones, así como acciones de vigilancia por parte de la Procuraduría Federal de Protección al Ambiente (PROFEPA) y la CONANP, que se limita a tres días de la semana en general.

- Bajo la tendencia actual se incrementará la presión de uso por visitantes (más de 300000 visitantes anuales).

- La capacidad de carga calculada ha sido rebasada ya con respecto al total de visitantes.

- Existen normas ambientales y turísticas diversas aplicables al manejo por parte de SEMARNAT y Secretaría de Turismo (SECTUR).

- Hay una inversión fuerte en la promoción, y prestadores de servicios que dependen del turismo en Islas Marietas.

- En el contexto nacional el turismo se considera una actividad económica de servicios prioritaria ante la caída de otros sectores.

- Se aproximan recortes presupuestales tanto a la SEMARNAT como a la investigación.

- Problemas de saturación de visitantes en días, horas y en sitios específicos como respuesta a la difusión espontánea y promoción dirigida.

- Ya hay un fuerte trabajo de regulación por diferentes instituciones.

- Existe participación de los diferentes sectores.

- Se necesitan ideas creativas y realistas para el manejo y conservación del ANP.

\section{PROBLEMÁTICA IDENTIFICADA Y JERARQUIZADA CON RESPECTO A LA VISITACIÓN EN EL PNIM}

Los principales problemas identificados -en primer lugar de importancia- fueron la alta afluencia de visitantes concentrados en sitios, temporadas y horarios, una inadecuada promoción como atractivo turístico 
donde el mensaje de área natural protegida no está implícito y las prácticas de los PST (Cuadro 2).

Cuadro 2. Problemática identificada y jerarquizada con respecto a la visitación

\begin{tabular}{|c|c|}
\hline Mesa 1 & Mesa 2 \\
\hline $\begin{array}{l}\text { 1. Picos de afluencia } \\
\text { 2. Embarcaciones informales } \\
\text { 3. Falta de vigilancia } \\
\text { 4. Promoción desmedida } \\
\text { inadecuada } \\
\text { 5. Poco respeto por parte de los PST }\end{array}$ & $\begin{array}{l}\text { 1. Promoción espontánea no } \\
\text { adecuada } \\
\text { 2. Uso de bloqueadores solares } \\
\text { 3. Modificación de hábitos } \\
\text { alimenticios de la fauna silvestre }\end{array}$ \\
\hline Mesa 3 & Mesa 4 \\
\hline $\begin{array}{l}\text { 1. Afluencia turística concentrada en } \\
\text { un mismo horario } \\
\text { 2. Desconocimiento de normas y } \\
\text { violación de reglamento } \\
\text { 3. Uso de bloqueador }\end{array}$ & $\begin{array}{l}\text { 1. Malas prácticas turísticas } \\
\text { 2. Se sobrepasa la capacidad de } \\
\text { carga diaria } \\
\text { 3. Libre acceso a la pesca } \\
\text { 4. No se vende correctamente el } \\
\text { destino } \\
\text { 5. Promoción desmedida e } \\
\text { inadecuada }\end{array}$ \\
\hline
\end{tabular}

Fuente: elaboración propia

\section{IDENTIFICACIÓN DE ESTRATEGIAS Y ACCIONES}

Una de las principales estrategias propuesta por los actores es la diversificación de los sitios de uso público, según sus puntos de vista esto disminuiría la presión que se ejerce sobre la Playa del Amor (Cuadro 3).

Cuadro 3. Resultados de las cuatros mesas de trabajo formato World Café

ZONIFICACIÓN

1. ¿¿Cómo funciona la zonificación actual del Parque? (Funcionalidad de la zonificación del Parque Nacional Islas Marietas, áreas, actividades permitidas y prohibidas, funcionalidad)

2. ¿Cómo funcionaría mejor? (Áreas propuestas, propuesta de actividades permitidas y prohibidas, funcionalidad deseable) 
Estrategias y acciones propuestas por los Actores

- $\quad$ Los PST no explican la zonificación del Parque.

- Concientizar al operador turístico sobre la zonificación del Parque.

- Desconocen por qué se denominó “área restringida” a la Playa del Amor.

- Cambiar tipo de uso de la Playa del Amor, de uso restringido a uso público.

- No recategorizar zona restringida a Playa del Amor.

- En la Playa del Amor cambiar zonificación a lo actual.

- Desconocen cuáles son las áreas prohibidas para la pesca.

- Ampliar la zona donde se restringe la pesca comercial.

- Establecer límites físicos para que se respete las zonas núcleo y zonas restringidas, por ejemplo, multiplicar el número de boyas para que las embarcaciones no se acerquen, o poner mallas para que los turistas no suban a la isla.

- Aumentar el número de boyas y colocarlas a una distancia mayor (fuera de las islas).

- Colocar boyas de amarre más efectivas, contrario a multiplicar el número debido a que estas requieren mantenimiento y el Parque no tendría que invertir en esto, además aumentaría el impacto a los corales.

- Ampliar zona de boyas en Playa del Amor para evitar riesgos.

- Elaborar reglamento de uso de boyas (boya para manejo y boya para señalización).

- Utilizar otro tipo de boyas o modificar las existentes para evitar aglomeraciones.

- Diseñar delimitación con boyado estratégico para que los operadores no encuentren la forma de pasar el límite de las líneas de boya.

- Delimitación (boyas) en el arco de la piedra.

- Señalamientos para negar acceso en el arco de la piedra a embarcaciones.

- $\quad$ Propone que se establezcan zonas de entrada y salida de embarcaciones para disminuir la carga de visitantes en una sola área.

- $\quad$ Establecer video-vigilancia para monitorear las embarcaciones que entran al Parque en horarios no permitidos, así como embarcaciones que pescan en áreas prohibidas.

- $\quad$ Establecer rastreo de embarcaciones turísticas y pesqueras (chip, GPS).

- Sistema de información geográfica para monitorear embarcaciones que no respeten zonas y horarios de uso del Parque.

- Ampliar la vigilancia las 24 horas del día los siete días de la semana (Video vigilancia)

- Monitoreo de embarcaciones por GPS.

- GPS más video vigilancia.

- Establecer horarios de visita de embarcaciones, rotando horarios por semana, dependiendo del tamaño de la embarcación.

- $\quad$ Establecer horarios de operación escalonado.

- Diversificar los sitios para uso público. 


\section{VISITACIÓN}

1. ¿Cómo se realiza la visitación al Parque? (Visitación, prestadores de servicios y particulares, situación actual, desarrollo de la actividad turística, usuarios, número de autorizaciones y espacios disponibles)

2. ¿Cómo podría realizarse mejor? (Cómo debería realizarse la visita, se debe promover la entrega de nuevas autorizaciones, criterios para sustentar la entrega o no entrega de más autorizaciones)

Estrategias y acciones propuestas por los actores

- Realizar una visitación selectiva.

- El visitante puede considerar que al tener una tarifa económica, no tiene valor ecológico, biológico o cultural.

- En la visitación establecer la información mínima que se debe otorgar a los visitantes con respecto al parque y su comportamiento para no sobrecargar de información al visitante

- Hacer hincapié en la importancia del lugar (sensibilización de los visitantes).

- Debe existir coordinación y logística entre los diferentes actores involucrados.

- Realizar una valorización del lugar.

- Reducción de basura de embarcaciones (Desechables).

- Que la embarcación cuente con radio (Capitanía de Puerto).

- Que se reporte a las autoridades de SECTUR, cuando el guía certificado cometa algún error, a fin de capacitarlos

- Revisiones de Capitanía de manera aleatoria.

- Creación de fundaciones para bajar recursos aplicables a ordenar la actividad en el área (ONG).

- Impuestos diferenciados por operador turístico, horas y días.

- Estudios econométricos.

- Establecer protocolos de información al visitante (habría que determinar cuándo y dónde).

- $\quad$ Establecer reglas de promoción del ANP.

- Elaborar y actualizar manuales dirigidos a visitantes.

- Establecer un sistema de reservaciones para la visita (aplicaciones, medios electrónicos).

- Creación de sustitutos de puntos críticos (Playa del Amor).

- Generar información en redes.

- Elaboración de anuncios.

- Promocionar el área especificando qué es un Parque Nacional.

- Se debe entregar información a los visitantes antes de embarcar.

- Certificar a los operadores turísticos en coordinación con instituciones.

- Uso de lenguaje asertivo en los materiales de información.

- Establecer cursos y requisitos para guías así como establecer las fechas de inicio. 
- $\quad$ Promover que los guías sean formales.

- Guías certificados en cada embarcación.

- Incluir un eslogan que eduque a los visitantes.

- Actualizar manuales y materiales de difusión en coordinación con Ayuntamientos.

- Utilizar las redes sociales para promocionar la información.

- Restringir las visitas fuera de horario.

- $\quad$ Promover que el uso del logo o la identidad del Parque tenga restricciones y obligaciones de uso solo para CONANP y SECTUR.

- Promover un sistema de reservación en los puntos críticos.

- Enviar a las diferentes instituciones información veraz para promover el área.

- Asegurar que todos los operadores presenten protocolos de operación.

- Realizar análisis de la operación de los tour operadores.

- La información se debe dar desde la contratación del tour.

- Crear sitios donde se promueva el área y se explique sus condiciones.

- Señalética de materiales alternativos (madera) de la Oficina de Visitantes y Convenciones/Asociación de Hoteles y Moteles de la Riviera Nayarit (OVC), de Puerto Vallarta y Ayuntamientos, y que los coloquen en playas y puntos de reunión y embarque.

- Mejorar los servicios ofrecidos sin bajar costos.

- Incluir en el análisis la visitación de población local y el cálculo de capacidad de carga.

- Creación de asociaciones y comités para operación y capacitación (asociaciones civiles, OVC, prestadores de servicios y ONG).

- Generar recursos para capacitaciones.

- Buscar donaciones.

- Seguimiento a la población flotante.

- Controlar los flujos de entrada y salida.

- Terminar con la problemática de conductos inapropiados como aglomeración, volumen, información incongruente.

- Debe existir una corresponsabilidad entre el operador y el guía para la capacitación, seguridad, conservación, manejo de grupos, contribuir con recursos, interpretación ambiental, entre otros a los que se deben sumar los prestadores de servicios y los Ayuntamientos.

- $\quad$ Se debe generar una cultura para tener visitantes informados.

- Buscar que los usuarios aporten recursos económicos.

- Generar nuevas alternativas de venta.

- Promover dos horarios de salida.

- Generar una política de obligaciones diferenciadas (costos a nacionales y extranjeros).

- Capacitación obligatoria con constancia de acreditación emitida por Ayuntamientos, Secretaría de Comunicaciones y Transportes (SCT) y CONANP entre otras. 
- Generar la reglamentación adecuada para el buen uso del área.

- Uso del área diferenciado de acuerdo a la capacidad y características de operación.

- Involucrar a promotores y agencias de viaje en la protección del área

- Valorar de manera adecuada el destino.

- Que los beneficios sean compartidos.

- Generar estrategias para que los prestadores cumplan con el pago de derechos.

- Asegurar que los servidores públicos cumplan y apliquen la reglamentación.

- Que el Gobierno del Estado promocione el área adecuadamente.

- $\quad$ Promover el estado del área adecuadamente por parte de los operadores.

- Mejorar las prácticas de los operadores turísticos.

- $\quad$ Establecer método para promover un mayor flujo de visitantes.

- Establecer carriles de entrada y salida.

- Sancionar a los guías que incumplan el reglamento.

- Buscar un distintivo para los operadores turístico que generen buenas prácticas.

- Involucrar a la SECTUR en la capacitación de los guías.

- Establecer mecanismos que aseguren que los guías conozcan la reglamentación.

- Promover la concientización desde el Gobierno del Estado.

- Promover elaboración de materiales.

- Utilizar materiales bien diseñados en base a un público directo, fácil de digerir y práctico.

- Promover que los pasaportes cuenten con fotografía.

- Promover mayor número de revisiones por parte de Capitanía.

- Buscar certificaciones locales a guías a través de SECTUR.

- Explicar la señalética y reglas de operación a los visitantes.

- Realizar convenios con las autoridades que puedan sancionar al operador turístico.

\begin{tabular}{|c|}
\hline REGLAMENTOS \\
\hline $1 . \quad \begin{array}{l}\text { ¿Qué falta en la reglamentación actual del Parque? (Capacidad de Carga } \\
\text { turística, quienes, cuántos, cuándo, dónde y cómo) }\end{array}$ \\
$\begin{aligned} & \text { 2. } \text { ¿Qué se debería agregar? (Límites de cambio aceptables, es necesario } \\
& \text { realizar cierres temporales, cuántos y que días, es posible realizar el } \\
& \text { sacrificio de zonas) }\end{aligned}$ \\
\hline Estrategias y acciones propuestas por los Actores \\
\hline - $\quad$ Disminuir el número de personas por embarcación de apoyo \\
- Toda promoción del Área Protegida debe llevar un slogan que resalte el ANP \\
Promover la modificación de la ley Federal de derecho para un cobro de \\
derechos diferenciado (Locales, Nacionales y Extranjeros)
\end{tabular}


- Colocación de boyas de amarre alejadas del sitio para la espera de las embarcaciones

- Que la empresa brinde alternativas como observación de aves, con guías de observación de aves y peces

- Prohibir el uso de desechables

- Que los prestadores de servicios enfaticen en que es un ANP e inmediatamente después se les coloque el brazalete a los visitantes

- Actualizar el estudio de Capacidad de Carga

- Revisiones en tierra de la capacidad de carga de una embarcación de manera aleatoria

- Solamente un Catamarán en la playa del amor

- Implementar turnos de acuerdo a la llegada (por radio)

- $\quad$ El último zarpe permitido a la visita de las Marietas será a las 17:00 horas desde Nuevo Vallarta

- Crear un Fideicomiso para la creación de un fondo operativo

- Concentrar las actividades en los sitios de alta visitación actual y limitar o restringir otras prioritarias

- Detener el tránsito de las embarcaciones ante la presencia de un mamífero (Ballenas)

- Utilización de sistemas de rastreo en embarcaciones

- $\quad$ Promover que el turismo sea selectivo

- Capacitación a PST (Buenas prácticas)

- Distribuir las visitas en diferentes sitios (No concentrar)

- $\quad$ Rotación de la visitación en sitios (Apertura y cierre de sitios)

- Establecer un "Hoy no circula” que podría ser: Días por puerto, días por tipo de embarcación u horarios por tipo de embarcación

- Limitar el tiempo de estancia

- Limitar el tipo de visitantes (Informar de los riesgos y que firmen una responsiva)

- A mediano y largo plazo ir introduciendo el tema de reservaciones

- Promover la vigilancia comunitaria y la autorregulación

- Turismo natural y cultural en lugar de turismo de aventura

\section{PARTICIPACIÓN SOCIAL}

1. ¿Qué capacitaciones se requieren para mejorar el desarrollo de la visitación en el Parque? (Temas, duración, instituciones que se han involucrado, cómo han sido las convocatorias)

2. ¿Qué hacer para aplicarlas? (A quién involucrar, cómo programarlas, qué mecanismos de acreditación se pueden implementar para acreditar a los operadores turísticos, costos y disponibilidad) 


\section{Estrategias y acciones propuestas por los actores}

- Los prestadores están conectados y se encuentran orgullosos de su actividad.

- Capacitación a los pescadores (trauma de los desplazados) están excluidos y no colaboran en el trabajo.

- Cumplir con las normas establecidas.

- Trabajo tripartito (gobierno-prestadores-sociedad).

- Delimitar atribuciones, competencias para una reacción positiva y común y trabajo integrado en conjunto que genere beneficios al prestador y a las personas.

- Capacitar a los prestadores, al gobierno, a las ONG y después a los turistas.

- $\quad$ Que se entienda que toda persona tiene derecho a buscar sustento.

- Guías capacitados, puede permitir que el número de visitantes se incremente.

- Capacitación de los promotores en lo referente a la sensibilización de los turistas.

- $\quad$ Plática previa con los turistas sobre el uso adecuado de bloqueador solar y el tipo adecuado (biodegradable, y aplicarlo 1 a 3 horas antes).

- $\quad$ Que exista un pre-registro con toda la información del área el cual tenga un costo para los PST

- Que los prestadores tomen un curso obligatorio y presenten un cartel con la información para sensibilizar a los turistas (el prestador debe dar la capacitación a los turistas).

- Guías de turismo de naturaleza.

- $\quad$ Promoción adecuada y real por parte del sector oficial.

- Que los prestadores cuenten con maquinistas especializados, que el guía sea certificado, que los propietarios cuenten con permiso y cumplan la norma.

- Pedir a cada capitán la libreta de navegación para verificar que se ha capacitado.

- Generar alianzas con otras instituciones, asociaciones y organizaciones gubernamentales.

- Realizar pláticas con los turistas al inicio del viaje sobre temas variados y amenos.

- Animación distinta, no solo centrada en diversión, baile y alcohol.

- Utilizar lenguaje de fácil comprensión.

- Actualización continua tanto de capacitadores como de prestadores de servicios turísticos.

- Firma de acuerdo con instituciones que se puedan involucrar en la capacitación.

- Que los prestadores estén obligados para renovar permisos a recibir la capacitación adecuada en cuanto al entendimiento del valor del ecosistema y las reglas de tensión al turista.

- Calendarizar y capacitar a todos los actores involucrados.

- Existen normas en turismo para los guías, pero existen muchos problemas de requisitos y burocracia. 
- Debe haber información con imágenes a la vista del público (display, con murales educativos en lugares públicos).

- Campañas de marketing verde y social que no lleve el propósito de compra o dañar sino más bien de conservar.

- Que exista mejor comunicación entre la capacitación de agentes de viaje y buscar opciones para la capacitación de funcionarios y prestadores.

- Capacitación para la sensibilización del aprovechamiento del área que no sea solo teórica sino también práctica y que genere una certificación con gafete.

- Capacitación en cuanto a la interpretación ambiental.

- Que exista señalización apropiada.

- Que las empresas grandes apoyen a las pequeñas en la capacitación para cuidar el ambiente, generando una asociación de conveniencia entre ambas.

- Diseño de productos turísticos diferentes, como museos o rutas.

- Generar distintivos a los prestadores de servicios que tengan buenas prácticas.

- SECTUR debería realizar la capacitación para los guías porque en la actualidad es muy cara para las empresas que contratan especialistas en los temas

- Realizar pláticas, charlas informativas en el puerto a la hora de embarcar.

- Paquetes turísticos diferentes que incluyan educación ambiental.

- Capacitación en reducción de basura.

- Incentivar las buenas prácticas entregando algún premio.

- Las instituciones de gobierno que se deben involucrar son SECTUR, SEMARNAT, CONANP, PROFEPA, Comisión Nacional de Acuacultura y Pesca, SCT (Capitanía de Puertos), Ayuntamientos de Bahía de Banderas y Puerto Vallarta, Gobierno del Estado Jalisco y Nayarit, Protección civil de ambos Estados (Nayarit y Jalisco) y, ONGs como PRONATURA entre otros.

- Generar incentivos para reforzar las buenas prácticas.

- Implementación de señalética por CONANP, Oficina de Visitantes y Convenciones y SECTUR

- Hacer volantes (guías de identificación de flora y fauna acuática) plastificados para su uso y venta.

- Capacitación de guías en manejo de residuos sólidos.

- $\quad$ Plática obligatoria en puerto de partida por parte de la empresa.

- Capacitación a guías locales en lo referente a turismo de naturaleza por parte del Estado y/o municipio con base a las normas federales, con reconocimiento local.

- Generar una certificación por parte de SECTUR con participación del Estado.

- Capacitar al turista desde los hoteles, comentarles que deben usar un brazalete y que visitarán un ANP.

- $\quad$ La CONANP debe coordinar las competencias entre los sectores.

- Marketing en que las agencias de viajes expliquen lo que es un ANP.

- Comerciales con imágenes de las ANP coordinados por CONANP y SECTUR. 
- Que el fideicomiso haga una promoción adecuada en cuanto a la difusión de la ANP.

- Levantar actas a los prestadores que realicen mal las prácticas.

- Alianzas para la capacitación EXPEDIA- UNESCO, tienen una alianza para la mejor comunicación del sitio y venta como sitio de patrimonio (paquetes capacitación para funcionarios y prestadores).

- Capacitar para la colaboración.

- Elaborar y entregar un volante informativo con las restricciones del área.

- Obligar a los prestadores a cumplir las reglas (buscar esquemas de sanción).

- $\quad$ Díptico de caricatura para fomentar valores y buen manejo del ANP.

Fuente: elaboración propia

\section{VISIÓN PARTICULAR POR ZONA}

De acuerdo al estudio técnico "Ordenamientos territoriales comunitarios y/o microrregionales: Programa de uso público del Parque Nacional Islas Marietas” (Biosfera, 2014), al registro diario de PST en campo por parte de la CONANP y a los talleres realizados en el World Café 2015 (mesa de trabajo Zonificación) con tomadores de decisiones, PST en La Cruz de Huanacaxtle y Puerto Vallarta, se elaboró la visión con afluencia de visitantes para las diferentes zonas y sitios en ambas islas (Cuadro 4), que establece la imagen del sitio a mediano plazo, con base a sus características actuales.

Cuadro 4. Visión por zona/sitio con base a características actuales y deseables. UP, Uso Público

\begin{tabular}{|l|l|l|}
\hline Sub-zona & Sitio & Características \\
\hline Isla Larga & $\begin{array}{l}\text { Cueva del } \\
\text { Muerto }\end{array}$ & $\begin{array}{l}\text { Zona de alto valor paisajístico marino y terrestre, } \\
\text { con gran variedad de peces e invertebrados que } \\
\text { pueden ser observados por buceo, donde se sigue } \\
\text { básicamente un sendero submarino y no se fractura } \\
\text { el coral ni se levanta sedimento. En la zona de } \\
\text { recuperación (R) establecida por investigadores } \\
\text { para monitoreo de crecimiento, el coral se recupera } \\
\text { y no se visita. Las embarcaciones respetan el } \\
\text { emboyado que delimita el área recreativa. Los } \\
\text { visitantes que realizan buceo libre utilizan } \\
\text { obligatoriamente el chaleco durante el tiempo que } \\
\text { permanecen en el agua. }\end{array}$ \\
\hline $\begin{array}{l}\text { UP2 } \\
\text { Isla Larga }\end{array}$ & La Nopalera \\
\hline
\end{tabular}




\begin{tabular}{|c|c|c|}
\hline & & $\begin{array}{l}\text { recreativo medio para natación, buceo libre y } \\
\text { autónomo, donde las embarcaciones respetan el } \\
\text { emboyado que delimita el área recreativa. }\end{array}$ \\
\hline \multirow[t]{4}{*}{$\begin{array}{l}\text { UP3 } \\
\text { Isla Redonda }\end{array}$} & $\begin{array}{l}\text { Pared } \\
\text { Paredón }\end{array}$ & $\begin{array}{l}\text { Sitio natural con paisajes rocosos y oquedades, } \\
\text { comunidades coralinas bien conservadas con } \\
\text { corales masivos y blandos de gran tamaño, pueden } \\
\text { observarse mantas, rayas y peces diversos.. }\end{array}$ \\
\hline & Túnel & $\begin{array}{l}\text { Sitio natural con colonias de corales (Porites) bien } \\
\text { conservadas con gran diversidad de peces y corales } \\
\text { blandos. }\end{array}$ \\
\hline & $\begin{array}{l}\text { Plataforma } \\
\text { Pavonas }\end{array}$ & $\begin{array}{l}\text { Sitio natural con comunidades coralinas donde } \\
\text { dominan las Pavonas. Gran cantidad de erizos y } \\
\text { peces. }\end{array}$ \\
\hline & Bajo rocoso & $\begin{array}{l}\text { Arrecife rocoso con gran cantidad de corales } \\
\text { blandos donde pueden observarse mantas y rayas. } \\
\text { Con baja frecuencia de uso. }\end{array}$ \\
\hline $\begin{array}{l}\text { UR1 } \\
\text { Isla Larga }\end{array}$ & $\begin{array}{l}\text { Sendero } \\
\text { terrestre }\end{array}$ & $\begin{array}{l}\text { Sitio prístino de muy baja intensidad de uso } \\
\text { recreativo, siempre un grupo pequeño con guía } \\
\text { certificado. Con acceso adecuado y señalización } \\
\text { pertinente. Los recorridos con un fuerte } \\
\text { componente de educación ambiental e } \\
\text { interpretación, donde se pueden observar aves } \\
\text { (bobos y gaviotas) anidando y criando. }\end{array}$ \\
\hline $\begin{array}{l}\text { UR2 } \\
\text { Isla Larga }\end{array}$ & $\begin{array}{l}\text { Escalones } \\
\text { de Piedra }\end{array}$ & $\begin{array}{l}\text { Sitio natural con comunidades coralinas bien } \\
\text { conservadas de bajo uso recreativo donde existe } \\
\text { una gran diversidad de peces e invertebrados. }\end{array}$ \\
\hline $\begin{array}{l}\text { UR3 } \\
\text { Isla Redonda }\end{array}$ & $\begin{array}{ll}\text { Playa del } \\
\text { Amor }\end{array}$ & $\begin{array}{l}\text { Sitio natural de belleza escénica significativa y uso } \\
\text { turístico alto, donde se recupera la cobertura de } \\
\text { coral. }\end{array}$ \\
\hline $\begin{array}{l}\text { Zona de } \\
\text { Amorti- } \\
\text { guamiento }\end{array}$ & General & $\begin{array}{l}\text { Sitio bien conservado donde se observan } \\
\text { mamíferos marinos. }\end{array}$ \\
\hline
\end{tabular}

\section{EL MANEJO DEL USO PÚBLICO EN EL PNIM}

A continuación, se describen las principales medidas que permitirían lograr la visión, las intensidades de uso y las clases de oportunidad antes descritas, así como, actividades en la zona de influencia que permita disminuir la presión en el Parque.

Actividades recreativas alternativas propuestas y sitios alternativos de uso: 
- $\quad$ Pajareando. Recorridos guiados para observación de aves desde embarcaciones utilizando binoculares y guías de aves. Dirigido a observadores de aves de Canadá y Estados Unidos. Solo en Estados Unidos existen alrededor de 55 millones de aficionados a la observación de aves que gastan en esta actividad aproximadamente 20 mil millones de dólares al año, más que los pescadores, cazadores o golfistas. En Canadá existe una situación similar (Del Olmo, 2009).

Senderismo en la Isla larga. Apertura del sendero establecido que concluye en la cámara de la cueva mayor. En este puede apreciarse la mayor parte del año a los pájaros bobos y gaviotas principalmente, sus nidos y polluelos entre enero y abril. Las cuevas y túneles se formaron por filtración de agua, erosión y acción del viento, están comunicados y abren ventanas a diversos paisajes de y desde la isla. Están asociadas a leyendas y costumbres locales.

- $\quad$ Arrecifes artificiales. El posicionamiento estratégico de estructuras de diversos materiales, acondicionadas para proveer hábitat y refugio a diversas especies de flora y fauna marina, en la zona de amortiguamiento o contiguo a la costa, permitiría disminuir la presión sobre arrecifes naturales, generar espacios para nueva vida submarina, educación e investigación; además de generar beneficios económicos a la población local por medio del ecoturismo. Puede disminuirse la pesca ilegal, crear fuentes de ingreso para el apoyo de otros programas y además fortalece la imagen del destino de sitio preocupado por la conservación.

- $\quad$ Parque submarino. Dedicado al arte, permitiría exhibir piezas de artistas de la región y, asociado a un "barco hundido", pueden funcionar como arrecifes artificiales generando los mismos beneficios.

- Atractivos relacionados con la pesca. La posibilidad de establecer criaderos de atún o la pesca con “almadraba” permite que visitantes incluso participen en las actividades $\mathrm{y}$, sobre todo esta última permite observar una gran cantidad de organismos además de peces, que pueden ser devueltos al mar. Esto podría hacerse fuera del Parque, pero asociado a las actividades pesqueras de Punta de Mita. 
- $\quad$ Playas artificiales. La recreación de una playa con las características de la Playa del Amor, es decir, un túnel que lleve a una playa localizada en una abertura circular y en una zona aislada no representa una obra ingenieril inalcanzable.

- Playa del Amor Virtual Expedition. El programa de realidad Virtual Expeditions que aloja Google permite a maestros y alumnos visitar y estudiar lugares en el mundo a los que no tienen acceso. Con fines educativos pueden crearse herramientas y paquetes educativos que estarían disponibles con la Cooperativa de Servicios Turísticos en Corral del Risco en un Centro de educación e interpretación ambiental y abierta en las redes solo como recorrido virtual.

Además, los PST establecieron que es el gobierno federal quien debe guiar dichas estrategias, en colaboración con las instituciones académicas. La mayoría de ellos debería ser cumplido en un corto plazo (3 meses).

\section{CONCLUSIONES}

En este estudio la herramienta de Café del Mundo ha permitido la inclusión de actores muy diversos que identifican como principal beneficio derivado del uso turístico del Parque Nacional Islas Marietas, el económico; en consecuencia las restricciones de visitación afectaría negativamente a los prestadores de servicios turísticos y sus familias; sin embargo, los mismo reconocen que las condiciones actuales que prevalecen en temporada alta provoca una mala experiencia para los visitantes con la posterior recomendación negativa a otros visitantes potenciales.

La problemática del uso público del Parque Nacional Islas Marietas está concentrada en cuanto al sitio utilizado, las actividades realizadas y los usuarios que las llevan a cabo. La principal actividad es la visita a la Playa del Amor a la cual se llega para realizar buceo libre o nadar, que es la actividad que genera los mayores impactos.

Entre la problemática detectada se encuentran que la investigación está fragmentada, la educación ambiental es incipiente, además existen conflictos entre los prestadores de servicios turísticos, investigadores en 
general y la administración del Parque y, la cantidad de visitantes excede a las capacidades de carga estimadas en el Parque. También se reconocieron que los principales impactos son debidos al hacinamiento en sitios visitados, la inmersión y la colecta de organismos.

Respecto a la experiencia de la visita se consideró mala o regular por diversos motivos; sin embargo, los visitantes están dispuestos a regresar. En cualquier caso, resulta indispensable el ordenamiento del uso público para la conservación del ecosistema, la satisfacción de los visitantes y, el bienestar de la comunidad local.

El buen manejo de las ANP depende en gran medida de la participación y la inclusión social aunque los beneficios se hacen evidentes generalmente de forma lenta y diferenciada. La participación social permite una toma de decisiones colectivas donde hay sacrificio de intereses particulares por los colectivos, no obstante, no existe garantía de que los intereses y aspiraciones de los involucrados terminen formando parte del documento final o, que siendo incluidas no formen parte del esquema de decisión final, por lo tanto, estarán ausentes las acciones que permitan cristalizar esas aspiraciones que derivan en acciones de beneficio social amplio. En este ejercicio participativo dominó el enfoque de conservación, donde algunos actores (los prestadores de servicios turísticos y pescadores) se consideraron como los de mayor influencia en la generación de los impactos negativos.

Así, la visión construida colectivamente del Parque es: Las actividades recreativas se realizan en las zonas permitidas con un fuerte componente de educación ambiental, donde todos los actores respetan las reglas y la capacidad de carga, misma que se establece de forma trasparente y colectiva; se monitorean los indicadores que determinan el límite de cambio aceptable, su aplicación y efectos y se involucra a todos los actores para que participen en estas tareas con el fin de asegurar la conservación del patrimonio natural, la continuidad de la visitación de las diversas áreas del Parque; así como, una alta calidad de la experiencia del visitante.

Estos talleres constituyeron el primer ejercicio que reunió a la mayoría de los actores involucrados en el uso y gestión del Parque, les permitió incluir sus opiniones y necesidades en un documento diagnóstico y generar estrategias, que en una nueva etapa del Parque, se encuentra en revisión. Posteriormente un grupo de ciudadanos, principalmente del sector turístico y que habían participado en este ejercicio descrito, 
preocupados por el futuro del Parque, se organizaran como asociación civil que denominaron "Bahía Unida” (2016) para defender sus derechos (ya que contaban con permisos vigentes de operar en el área) ante el cierre de la Playa del Amor en el 2016 (CONANP, 2016). Otro fin de dicha asociación que se establece como prioritario, es innovar en la colaboración en tareas como la conservación y capacitación. Además, la cooperación con ONG y entidades gubernamentales se ha incrementado notablemente. Esto hace un ejemplo único en México de gestión de un área natural que aún tiene muchos retos, pero ha conseguido logros importantes en la conservación de forma participativa.

Los ecosistemas insulares costeros constituyen una forma de interacción fundamental entre la zona litoral, marina y terrestre, que condicionan la vida de varias especies terrestres y marinas, especialmente de aves migratorias y residentes; así como, de las comunidades coralinas. Estos procesos dan valor paisajístico y escénico que también deben permanecer. Esto requiere de un manejo de recursos y gestión del uso público consensuado, bajo medidas técnicas que mantengan o creen las condiciones biofísicas, socioculturales y administrativas deseables sin rebasar los límites establecidos.

\section{REFERENCIAS}

Bahía Unida (2016). Bahía de Banderas. Bahía A.C. http://www.bahiaunida.org/

Biosfera (2014). Programa de conservación para el desarrollo sostenible 2014, Parque Nacional Islas Marietas. Informe del Estudio técnico Ordenamientos territoriales comunitarios y/o microrregionales. Programa de Uso Público del Parque Nacional Islas Marietas. Puerto Vallarta, Jalisco, México.

Borrini-Feyerabend, Grazia; Dudley, Nigel; Jaeger, Tilman; Lassen, Barbara; Pathak Broome, Neeman, Phillips, Adrian. \& Sandwith, Trevor (2013). Governance of protected areas: from understanding to action. Best practice protected area guidelines. Series no. 20. IUCN, Gland, Switzerland. [online]. https://www.iucn.org/about/work/programmes /gpap_home/gpap_capacity2/gpap_bpg/?13678/Governanceo f-Protected-Areas-From-understanding-to-action 
Brown, Juanita \& Isaacs, David (2005). The World Café Community. The World Cafe: Shaping Our Futures Through. San Francisco: Bernett-Koehler.

Calvet-Mir, Laura; Maestre-Andrés, Sara; Molina, José Luis \& van den Bergh, Jeroen (2015). Participation in protected areas: a social network case study in Catalonia, Spain. Ecology and Society 20(4):45. http://dx.doi.org/10.5751/ES-07989-200445 Comisión Centroamericana de Ambiente y Desarrollo (CCAD), Sistema Arrecifal Mesoamericano (SAM) (2005). Compiladores: Arellano-Guillermo Alfredo, Alvarez Gil, Oscar, García-Rivas Maria del Carmen y Jeanett Acosta-Aburto. Manual de Métodos para la Elaboración de Programas de Uso Público en Áreas Protegidas de la Región del Sistema Arrecifal Mesoamericano. Belice: Mesoamerican Barrier Reef Systems Project. www.mbrs.doe.gov.bz/dbdocs/tech/es_PubUse.pdf

Comisión Nacional de Áreas Naturales Protegidas (CONANP) (2005). Programa de conservación y manejo, Parque Nacional Islas Marietas, México. México, D.F.

Comisión Nacional de Áreas Naturales Protegidas (CONANP) (2016). La CONANP anuncia cierre de la playa del amor. Comunicado de prensa CONANP/SEMARNAT No.

31. http://www.conanp.gob.mx/difusion/comunicado.php?id subcontenido $=1003$

Cornejo-Ortega, José Luis; Chávez-Dagostino, Rosa María \& CupulMagaña, Amilcar (junio 2011). Estimating carrying capacity in a natural protected area as a conservation strategy. Conference abstracts. En: 31st Annual Meeting of the International Association for Impact Assessment. Impact Assessment and Responsible Development. 29 may-4 jun. Final Program, Abstracts Posters, p.41. http://conferences.iaia.org/2011/pdf/web-final-programiaia11.pdf

Cornejo- Ortega, José Luis \& Chávez- Dagostino, Rosa María (2014). La huella de carbono de la observación de ballena jorobada (Megaptera novaeangliae) en las islas Marietas, Nayarit, México. Rev. Int. Contam. Ambie., 30 (1): 121-130.

Cornejo-Ortega, José Luis; Chávez-Dagostino, Rosa María \& CupulMagaña, Fabio Germán (2016). Éxito reproductivo de los 
pájaros bobos patas azul, Sula nebouxii, y los pájaros bobos café, Sula leucogaster, como indicador de perturbación por uso turístico en las Islas Marietas, México. Animal Biodiversity and Conservation, 39 (2):185-193.

Cupul-Magaña, Amilcar Levi \& Rodríguez-Troncoso, Alma Paola (2017). Tourist carrying capacity at Islas Marietas National Park: An essential tool to protect the coral community. Applied Geography, 88: 15-23.

Del Olmo, Gerardo (2009). Manual para Principiantes en la Observación de las Aves. México, D.F. Bruja de Monte.

Espinosa, Mario (2009). La participación ciudadana como una relación socio-estatal acotada por la concepción de democracia y ciudadanía. Andamios, 5 (10): 71-109

Gaviño, Gonzalo \& Uribe, Z. (1981). Distribución, población y época reproductiva de las aves de las islas Tres Marías, Jalisco. Anales del Instituto de Biología, Serie Zoología, 51: 505-524.

Halffter, Gonzalo (1984). Reservas de la Biosfera: Conservación de la Naturaleza para el hombre. Acta Zoologica Mexicana, (ns) 5: 4-48.

Londoño, J. M. (2017). Ecoturismo: Estrategia de Conservación de las Áreas Naturales Protegidas. Latinoamérica al Natural. Redparques 2: 3-4.

Naughton-Treves, Lisa; Buck Holland, Margaret \& Brandon, Katrina (2005). The role of protected areas in conserving biodiversity and sustaining local livelihoods. Annu. Rev. Environ. Resour., 30 (1): 219-252.

Patten, Chris (2000). Governance. Reith Lectures 2000: Respect for the Earth. 1: Governance. Recuperado de http://downloads.bbc.co.uk/rmhttp/radio4/transcripts/2000 0412_reith.pdf

Paz Salinas, María Fernanda (2008). De áreas naturales protegidas y participación: convergencias y divergencias en la construcción del interés público. Nueva antropol. [online], 21 (68):51-74.

Preciado, Rita Marcela; Simental, Ricardo; Ochoa-Chávez, José Miguel \& Chávez-Dagostino, Rosa María (2011). Consideraciones para 
la evaluación del impacto ambiental del buceo recreativo en el Parque Nacional Islas Marietas. En E. Andrade, R. M. Chávez-Dagostino y R. Espinoza (Eds), Turismo, Desarrollo y Región Estudio de Casos (pp. 59-78). Guadalajara: Prometeo/ Universidad de Guadalajara.

Riemann, Hugo; Santes-Álvarez, Ricardo V. \& Pombo, Alberto (2011). El papel de las áreas naturales protegidas en el desarrollo local: El caso de la península de Baja California. Gestión y Política Pública, 20 (1): 141-172.

Rodríguez Quiroz, G. \& Bracamonte Sierra, A. (2008). Pertinencia de las ANP como política de conservación y mejoramiento de la calidad de vida. Análisis de percepción en la Reserva de la Biosfera del Alto Golfo de California y Delta del Río Colorado. Estudios Sociales, 16 (32): 8-36.

Ruíz-Mallén, Isabel; De la Peña, Antonio; Méndez-Lopez, María Elena \& Porter-Bolland, Luciana. (2013). Local participation in community conservation: methodological contributions: 117133, in Luciana Porter-Bolland, Isabel Ruíz-Mallén, Claudia Camacho-Benavides, and Susanaha R. McCandless (editors). Community action for conservation: Mexican experiences. Springer, Berlin, Germany. http://dx.doi. org/10.1007/978-14614-7956-7_8

Wesselink, Anna; Paavola, Jouni; Fritsch, Oliver \& Renn, Ortwin. (2011). Rationales for public participation in environmental policy and governance: practitioners' perspectives. Environment and Planning A 43:2688-2704. http://dx.doi.org/10.1068/a44161

Wyrtki, Klaus (1965). Surface currents of the Eastern Tropical Pacific Ocean. Inter-American Tropical Tuna Commission Bulletin, 9 (5): 268-305. 